\title{
Análisis psicopatológico y sociodemográfico de pacientes hospitalizados por intento de suicidio en un hospital público chileno: estudio transversal analítico Psychopathological and sociodemographic analysis of hospitalized patients for suicide attempt in a Chilean public hospital: An analytical cross-sectional study
}

Rebeca Núñez ${ }^{\mathrm{a}}$, Marcelo Arancibia ${ }^{\mathrm{b}}$, Reginald Rees ${ }^{\mathrm{a}}$

\begin{abstract}
Introduction: Suicide is a global public health problem. At the regional level, the analysis of the psychopathological and sociodemographic dimensions of suicide attempt (SA) are scarce. Methods: We conducted a cross-sectional study to analyze psychopathological and sociodemographic characteristics of adults hospitalized for $S A$ in a public hospital in Chile. We describe participants according to sociodemographic and clinical characteristics, appraising neuropsychiatric syndromes, personality disorders, and levels of hopelessness, impulsiveness, intentionality, lethality and aggressiveness of the SA. Non-parametric statistics were applied. Results: 45 participants were included, with a median of 39 years of age. The majority lived in urban areas, belonged to low socioeconomic status, were single, had some stressful life event and a history of SA. The most frequent SA method was drug intake. The most frequent diagnoses were alcohol use disorder and borderline personality. In half of the cases the hopelessness was low or inexistent. Lethality was positively and significantly correlated with suicidal intent, as was impulsivity with aggressiveness. Family history of SA and psychological trauma were significantly higher in women and chronic pain in men. Severe cases showed high levels of hopelessness, intentionality, and lethality, while impulsivity was significantly less than in non-severe cases. In this group, the most frequent diagnosis was depressive episode. Conclusions: The analyzed sample showed distinctive psychopathological and sociodemographic characteristics. The lethality and intent of the SA must be systematically appraised. The severe cases of SA showed particular psychopathological characteristics.
\end{abstract}

Keywords: suicide, psychopathology, adult, hospitalization.

Rev Chil Neuro-Psiquiat 2021; 59 (1): 2-15

Los autores declaran no tener conflictos de intereses.

Aceptado: 2020/08/06

Recibido: 2020/04/14

a Departamento de Psiquiatría, Escuela de Medicina, Universidad de Valparaíso,

Valparaíso, Chile.

Centro Interdisciplinario de Estudios en Salud (CIESAL), Escuela de Medicina, Viña del Mar, Chile 


\section{INTRODUCCIÓN}

E suicidio ha experimentado un aumento en los últimos 50 años, transformándose en un problema de salud pública global ${ }^{1}$. Según datos de la Organización Mundial de la Salud, las muertes por este fenómeno podrían llegar a 1,5 millones durante 2020 , convirtiéndose en la segunda causa de muerte en la población entre 15 y 29 años ${ }^{2}$. En la región de Valparaíso, Chile, registros del Servicio de Salud Viña del Mar- Quillota muestran 437 casos de intentos de suicidio (IS) en hospitales de la $\mathrm{red}^{3}$, cifra que se duplica en 2015, llegando a 969 casos ${ }^{4}$, coincidente con lo observado a nivel nacional.

La suicidalidad es un espectro que va desde la ideación, las comunicaciones y los comportamientos asociados al IS, hasta el suicidio consumado. Esta diferenciación conceptual resulta importante al momento de revisar la frecuencia de cada una. Por ejemplo, por cada suicidio consumado al menos 20 personas realizan un IS $^{2}$. Este fenómeno difiere según sexo, grupos etarios, regiones geográficas y escenarios sociopolíticos, lo que sugiere una etiología heterogénea y multicausal ${ }^{5}$. Por lo tanto, el conocimiento de factores individuales y sociales es imperativo en la comprensión global del riesgo suicida. En efecto, existe evidencia de ciertos factores de riesgo para la conducta suicida, tales como características psicopatológicas, eventos vitales estresantes y determinantes sociodemográficos ${ }^{6}$. Aunque clásicamente la literatura ha vinculado a la gravedad del IS parámetros tales como la presencia de un desorden psiquiátrico, el antecedente familiar o individual de IS y/o el acceso a métodos letales, los factores sociodemográficos, altamente diversos según la muestra estudiada, resultan igualmente importantes, tales como la presencia de redes de apoyo, de patologías crónicas, la edad, la posición laboral y el nivel económico ${ }^{7}$. Lo mismo sucede con características psicopatológicas que no suelen evaluarse en servicios de urgencia que no cuentan con médicos especialistas, tales como la desesperanza ${ }^{8-10}$ y la impulsividad ${ }^{11}$. Todos ellos son de gran utilidad en la evaluación del riesgo suicida de pacientes que consultan en atención de urgencias, punto crítico de identificación e intervención de la suicidalidad en pacientes de alto riesgo. En efecto, se ha corroborado que una gran proporción de las personas que mueren por suicidio han sido atendidas en un servicio de urgencias previamente al deceso ${ }^{12}$. Un estudio de cohorte realizó un seguimiento de cinco años a un grupo de 302 pacientes hospitalizados por IS de alta gravedad médica; se estimó que 37\% de ellos realizó por lo menos un nuevo IS y $6,7 \%$ murió por suicidio ${ }^{13}$. Esto confirma además que las personas que realizan un IS de mayor gravedad pueden presentar características específicas. No obstante, el perfil de pacientes que consultan por IS ha sido escasamente descrito a nivel regional e incluso nacional, información que permitiría una mayor prevención y un mejor manejo $^{12,14}$. Por lo tanto, el objetivo de esta investigación es caracterizar psicopatológica y sociodemográficamente a pacientes consultantes por IS en un hospital de Viña del Mar, Chile, identificando a los casos más graves y analizando las asociaciones entre distintos parámetros de esta naturaleza.

\section{MÉTODOS}

\section{Muestra}

Se realizó un estudio transversal con orientación analítica para caracterizar psicopatológica y sociodemográficamente a los pacientes hospitalizados por IS en la Unidad de Psiquiatría Intrahospitalaria del Hospital Dr. Gustavo Fricke de Viña del Mar, Chile. La muestra quedó conformada por todos los pacientes que consultaron 
entre diciembre de 2017 y febrero de 2018 en la Unidad de Emergencia de Adultos, con diagnóstico de ingreso de IS según la definición de Silverman et a ${ }^{15}$, de 18 años o más y estables en su condición médica.
Se excluyó a los pacientes con alteración de conciencia. Dos psiquiatras de adultos y una psicóloga evaluaron a cada paciente durante tres días. Los instrumentos aplicados se puntualizan en Tabla 1.

Tabla 1. Descripción de instrumentos.

\section{Cuestionario de antecedentes sociodemográficos y clínicos}

Cuestionario de elaboración propia que recopiló antecedentes sociodemográficos (edad, sexo, nacionalidad, lugar de residencia, estado civil, grupo familiar, ocupación, nivel educacional, nivel socioeconómico, pueblo originario de identificación, pertenencia a una iglesia, presencia de estrés familiar y/o sociolaboral) y clínicos (presencia de enfermedades crónicas y/o terminales, síndromes dolorosos y/o discapacidad, antecedentes individuales y familiares de IS, antecedente de trauma psíquico y método del IS). El cuestionario se administró en el contexto de una entrevista psiquiátrica semi-estructurada.

\section{Escala de Intencionalidad Suicida de Beck (Suicide Intent Scale, SIS) ${ }^{(16)}$.}

Escala heteroaplicada, ampliamente usada en la evaluación del riesgo suicida. La SIS valora la seriedad o intensidad con la que alguien intentó suicidarse y sobrevivió al intento. Consta de 20 ítems que cuantifican la conducta verbal y no verbal del intentador durante el más reciente IS. Cada ítem se puntúa en una escala de 0 a 2 con un puntaje total de 0 a 30 . La primera parte (ítems 1-8) evalúa las circunstancias objetivas del IS e incluye ítems respecto a la preparación y la ejecución del intento. La segunda parte (ítems 9-15) evalúa la percepción de letalidad del método que el intentador posee, expectativas acerca de la posibilidad de rescate e intervención. La tercera parte (ítems 16-20) evalúa las circunstancias subjetivas. Esta escala genera tres categorías según gravedad: leve (0-7 puntos), moderada (8-13 puntos) y alta (14 o más puntos). La SIS es una herramienta ampliamente usada en la evaluación del riesgo suicida.

Escala de Letalidad de Beck (Beck Lethality Scale, BLS) ${ }^{(17)}$. Escala heteroaplicada que mide la letalidad de un IS en personas que han presentado uno recientemente. El puntaje de daño médico va desde 0 (completamente conciente y alerta) a 10 (muerte). El puntaje total, de 0 a 10, está basado en el examen físico del paciente y su grado de compromiso al momento de la admisión al servicio médico. Esta escala estima tres niveles de letalidad: baja (0-1 puntos), moderada (2-3 puntos) y alta (4-10 puntos).

Cuestionario de Agresividad de Buss y Perry (Buss-Perry Agression Questionnaire, BPAQ ${ }^{(18)}$. Corresponde a una revisión actualizada del Cuestionario de Hostilidad de Buss-Durkee, en donde se incorporan los componentes motores, cognitivos y afectivos del constructo agresividad, considerándose como el estándar de referencia para su medición. El cuestionario consiste en 29 ítems autoadministrados, valorados según una escala de 5 puntos. El BPAQ incluye cuatro subescalas: "agresión física", "agresión verbal”, "ira” y "hostilidad". El puntaje de cada escala es la suma de los ítems, mientras que el puntaje total de agresión es la suma de estas escalas. El instrumento cuenta con versiones validadas al castellano que señalan que su consistencia interna es adecuada ${ }^{(19,20)}$. 
Escala de Impulsividad de Barratt-11 (Barratt Impulsivity Scale, BIS-11) ${ }^{(21)}$. Escala autoaplicada de 30 preguntas que evalúan impulsividad en el actuar y el pensar. Cada respuesta hace alusión a la frecuencia: "raramente o nunca", "ocasional", "a menudo", "siempre o casi siempre". Se puntúa mediante una escala Likert que va de de 0 a 4 . Esta escala se compone de 3 factores independientes: impulsividad cognitiva, impulsividad motora y ausencia de planificación. Sin embargo, se suele resumir en una única puntuación total que se obtiene sumando los ítems individuales. Esta escala resulta en tres niveles de impulsividad: ninguno (0-51 puntos), normal (52-71 puntos) y alto (72 o más). La BIS-11 se encuentra validada al castellano ${ }^{(22)}$.

Escala de Desesperanza de Beck (Beck Hopelessness Scale, BHS) ${ }^{(23) .}$ Escala autoaplicada que consta de 20 ítems con declaraciones verdaderas y falsas diseñadas para evaluar las creencias positivas y negativas acerca del futuro y de sí mismo durante la última semana. Estos 20 ítems son puntuados como " 0 " o " 1 ", dependiendo si la respuesta es falsa o verdadera. El puntaje total va a de 0 a 20 . Esta escala genera cuatro categorías según el nivel de desesperanza: ninguno (0-3 puntos), leve (4-8 puntos), moderado (9-14 puntos) y alto (15-20). Se utilizó la versión del instrumento validada al castellano para pacientes con riesgo suicida ${ }^{(24)}$.

Escala de la Evaluación Cognitiva de Montreal (Montreal Cognitive Assessment, MoCA ${ }^{(25)}$. Instrumento que evalúa la función ejecutiva y visoespacial, identificación, memoria, atención, lenguaje, abstracción, recuerdo y orientación. La validación al castellano realizada en Chile indica que un puntaje $<21$ tiene el mayor rendimiento para deterioro cognitivo leve (sensibilidad de $75 \%$ y especificidad de $82 \%)^{(26)}$.

MINI Entrevista Neuropsiquiátrica Internacional 7.0 (MINI International Neuropsychiatric Interview, MINI) ${ }^{(27)}$. Corresponde a una entrevista estructurada breve para enfermedades neuropsiquiátricas que se usó para la estandarización de los diagnósticos. Está dividida en módulos identificados por letras que corresponden a una categoría diagnóstica. Al comienzo de cada módulo diagnóstico existen preguntas de tamizaje correspondientes a los principales criterios de la patología. Todas las preguntas deben ser calificadas como "Sí" o "No". Los criterios no deben ser calificados como positivos cuando los síntomas pueden ser causados por consumo de sustancias o por alguna causa médica de base. Por lo tanto, el criterio del clínico que ejecuta la entrevista debe primar para calificar las preguntas.

Cuestionario de Evaluación IPDE módulo DSM-IV (International Personality Disorder Evaluation Screening Questionnaire, IPDE-SQ) ${ }^{(28)}$. Cuestionario autoaplicado que identifica aquellos rasgos y conductas relevantes para la evaluación de los criterios de trastornos de personalidad según el sistema de clasificación del Manual Diagnóstico y Estadístico de los Trastornos Mentales en su $4^{\mathrm{a}}$ Versión (DSM-IV). Estos aspectos deben estar presentes en personas adultas y mantenerse durante los últimos cinco años. Consta de 77 ítems con respuestas que se califican como verdadero o falso. Las respuestas verdaderas se puntúan con " 1 ” y las falsas con " 0 ". Existen ítems que no son aplicables a algunos sujetos y son puntuados como "No aplicable". Si la persona puntúa más de tres puntos para un tipo de trastorno de personalidad, es necesario aplicar la entrevista semi-estructurada completa. Los resultados se categorizan en 10 trastornos de personalidad expuestos en el DSM-IV. La entrevista no debe aplicarse en pacientes con agitación severa, psicosis, discapacidad intelectual o deterioro cognitivo. El instrumento cuenta con una versión validada al castellano ${ }^{(29)}$. 
El IPDE-SQ no fue aplicado en los casos en que se pesquisó algún trastorno psicótico o un deterioro cognitivo de causa médica (MINI). En este caso, se administró la MoCA para precisar el diagnóstico. Los participantes que obtuvieron niveles altos en SIS y BLS se categorizaron como "casos graves".

\section{Análisis estadístico}

Para el análisis descriptivo se utilizaron medianas, rangos intercuartílicos (RIQ) y proporciones. En el análisis inferencial se utilizó la prueba de Wilcoxon-MannWhitney para comparar medianas y la prueba exacta de Fisher para comparar proporciones. Se realizó una matriz de correlación de variables cuantitativas mediante la prueba de correlación de Spearman. Se utilizó un nivel de significancia del 5\%. Los datos fueron analizados con el programa estadístico Stata 16 (StataCorp, Texas, Estados Unidos). El reporte de este estudio se ajusta a la pauta STROBE para estudios observacionales ${ }^{(30)}$.

\section{Aspectos bioéticos}

Todos los participantes siguieronun protocolo de consentimiento informado. El estudio fue evaluado y aprobado por el Comité de Bioética de la Facultad de Medicina de la Universidad de Valparaíso, Chile (Acta de Evaluación Número 40/2017).

\section{RESULTADOS}

\section{Muestra total}

La muestra se conformó por 45 participantes, con una mediana de edad de 39 años (RIQ 29-52). El 64,4\% fueron mujeres $(n=29)$, casi la totalidad fueron chilenos $(97,7 \%)$ y vivía en zonas urbanas $(95,5 \%)$. Un $40 \%(n=18)$ se encontraba soltero, un $20 \%(\mathrm{n}=9)$ casado, mientras que los viudos y los convivientes fueron $11,1 \%(n=5)$ en cada caso. El tipo de familia más frecuente fue la nuclear $(33,3 \%$; $\mathrm{n}=15)$, destacando luego un 26,6\% $(\mathrm{n}=12)$ que vivía como allegado y un $13,3 \%(\mathrm{n}=6)$ que vivía solo. Cerca de la mitad (46,6\%) contó con estudios secundarios, mientras que $31,1 \%(n=14)$ solo presentaba estudios primarios; una minoría $(11,1 \% ; n=5)$ había cursado estudios superiores. En cuanto a la ocupación, un tercio se encontraba empleado $(n=15)$, un tercio desempleado $(n=15), 7$ recibían una jubilación, 5 fueron dueñas de casa y 3 estudiantes. Cerca de la mitad de la muestra se encontraba en el segundo quintil de ingresos económicos $(48,8 \% ; n=22)$, es decir, percibía una renta mensual de 86 a 145 USD. La mayoría no se identificó con algún pueblo originario $(95,5 \% ; n=43)$ o con algún credo religioso $(53,3 \% ; n=24)$, pero la Iglesia de filiación predominante fue la católica $(33,3 \% ; n=15)$. Casi la totalidad de la muestra exhibió algún evento vital estresante $(n=44)$, siendo el más frecuente el asociado a los conflictos de pareja (60\%; $\mathrm{n}=27$ ).

Los métodos de intento suicida correspondieron a ingesta medicamentosa $(66,6 \% ; \quad n=30), \quad$ ahorcamiento $\quad(13,3 \%$; $\mathrm{n}=6)$, ingesta de otras sustancias $(8,8 \%$; $\mathrm{n}=4)$, heridas cortopunzantes $(6,6 \% ; n=3)$, inmersión $(2,2 \% ; n=1)$ y precipitación $(2,2 \% ; n=1)$. Un 55,5\% $(n=25)$ presentó el antecedente de algún tipo de trauma psíquico y un $68,8 \%(n=31)$ ya contaba con un IS.

Los resultados de los instrumentos aplicados a la totalidad de la muestra, la matriz de correlación entre los puntajes obtenidos y la comparación según sexo se resumen en las siguientes tablas. 
Tabla 2. Resultados de SIS, BLS, BPAQ, BIS-11, BHS, MINI e IPDE-SQ de la muestra total.

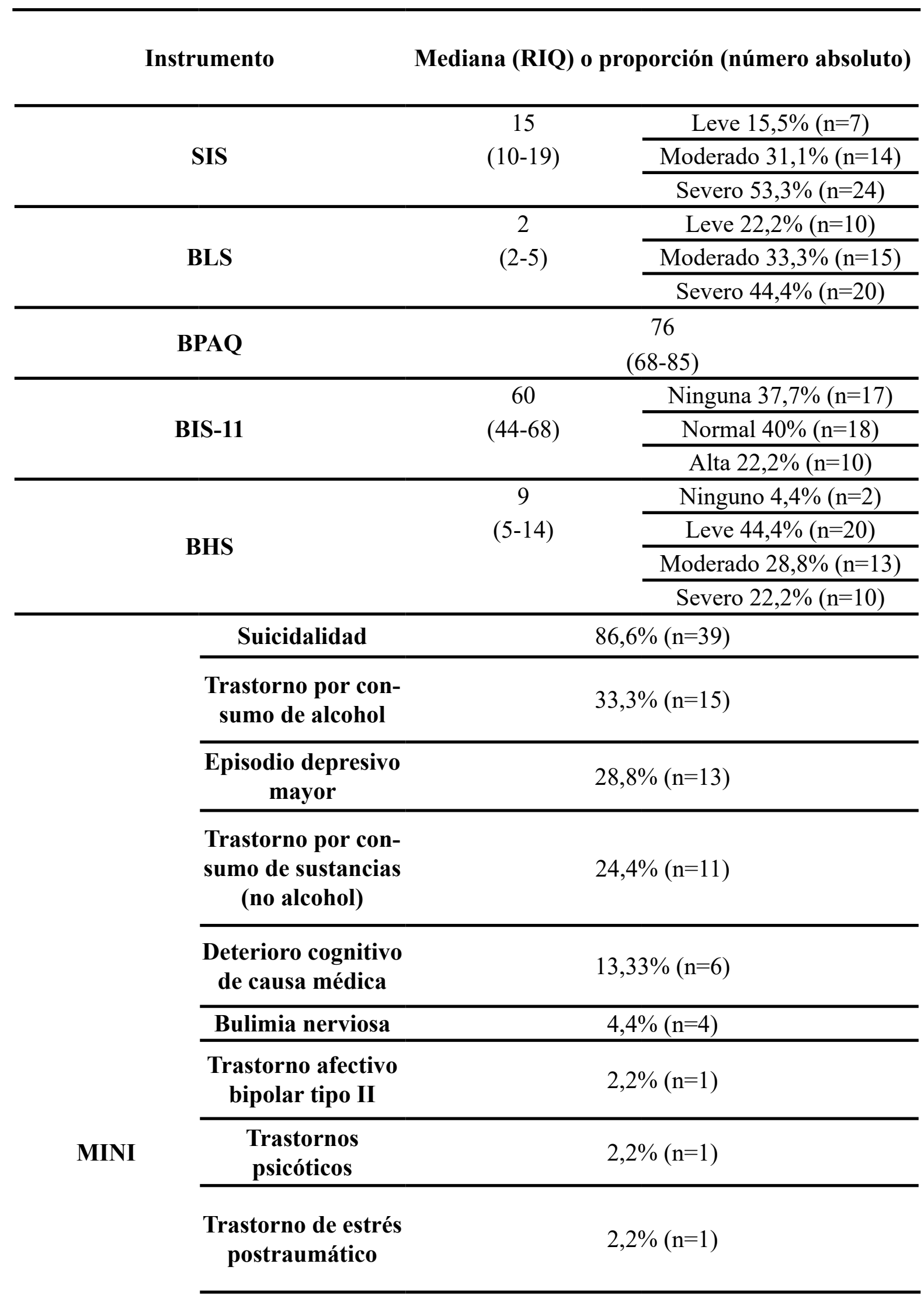




\begin{tabular}{cc}
\hline $\begin{array}{c}\text { Trastorno de } \\
\text { Pánico }\end{array}$ & $2,2 \%(\mathrm{n}=1)$ \\
\hline Límite & $44,7 \%(\mathrm{n}=17)$ \\
\hline Ninguno & $42,1 \%(\mathrm{n}=16)$ \\
\hline Antisocial & $7,8 \%(\mathrm{n}=3)$ \\
\hline Narcisista & $2,6 \%(\mathrm{n}=1)$ \\
\hline Evitador & $2,6 \%(\mathrm{n}=1)$ \\
\hline
\end{tabular}

El IPDE-SQ no se aplicó en un participante que resultó con diagnóstico de trastorno psicótico y en seis participantes con deterioro cognitivo de causa médica. En estos últimos seis casos se aplicó el MoCA, exhibiendo $5 / 6$ participantes un resultado compatible con deterioro cognitivo leve. (tabla 3 y tabla 4).

\section{Casos graves}

Los casos más severos, determinados según intencionalidad y letalidad, correspondieron a 11 participantes; la mediana de edad fue de 41 años (RIQ 31-68), 54,5\% $(n=6)$ fueron mujeres y $45,4 \%(n=5)$ tuvieron antecedentes de IS. En 72,7\% ( $\mathrm{n}=8)$ de los casos el método fue la ingesta medicamentosa. Un $81,8 \%(\mathrm{n}=9)$ no tenía un empleo actual y de ellos dos fueron estudiantes, mientras que un $90,9 \%(n=10)$ no tuvo pareja. El diagnóstico psiquiátrico más frecuente fue episodio depresivo mayor $(54,5 \%$; $n=6)$, seguido de trastorno por consumo de alcohol $(36,3 \%$; $\mathrm{n}=4)$ y deterioro cognitivo leve $(36,3 \%$; $\mathrm{n}=4)$. En Tabla 5 se presenta un análisis comparativo según gravedad del IS.

\section{DISCUSIÓN}

El estudio se conformó por participantes con una mediana de 39 años de edad, 64\% mujeres, que mayormente residía en zonas urbanas y pertenecía a un nivel socioeconómico bajo. Casi la totalidad presentó algún evento estresante, la mitad tenía antecedente de trauma psíquico y $68,8 \%$ de IS.
Se ha observado que en países desarrollados y en vías de desarrollo el sexo femenino sería un factor de riesgo para el $\mathrm{IS}^{(31)}$. A su vez, los antecedentes de IS familiar y de trauma psíquico fueron significativamente mayores en las mujeres $(p=0,004)$, factores de riesgo de suicidio consistentemente informados por la literatura. Asimismo, la distribución por sexo pudo haberse relacionado con que el método de IS más usado fue la ingesta medicamentosa $(66,6 \%)$, medio de mayor uso entre las mujeres ${ }^{(32)}$.

Por otra parte, más de la mitad de la muestra no tenía pareja durante el IS, aspecto controvertido en la literatura. Algunos modelos estadísticos que controlan por factores de confusión han demostrado que el estado civil no influiría significativamente sobre el IS $^{(33)}$, mientras que otros afirman que el tener una relación de pareja sería un factor protector para el suicidio, por proveer de una red de protección y apoyo. No obstante, la viudez y el haber terminado una relación de pareja recientemente sí aumentarían la probabilidad de IS (estresores vitales) y no necesariamente el hecho de estar soltero, como lo demuestra un estudio prospectivo que reclutó a 47.604 participantes $^{(34)}$. Nuestros resultados también exhiben que casi todos los participantes presentaban al menos un evento vital estresante, siendo los más frecuentes los conflictos interpersonales, sobre todo los conflictos de pareja, observados en aproximadamente un $60 \%$ de los pacientes. Esto podría asociarse a la alta frecuencia de desórdenes de la personalidad, como se de- 
Tabla 3. Matriz de correlación entre puntajes totales de SIS, BLS, BPAQ, BIS-11 y BHS.

\begin{tabular}{cccccc}
\cline { 2 - 6 } SIS & SIS & BLS & BPAQ & BIS-11 & BHS \\
\hline \multirow{2}{*}{ BLS } & 1 & & & & \\
& $*, 391$ & 1 & & & \\
\hline \multirow{2}{*}{ BPAQ } & $0,003(0,007)$ & - & & & \\
& & $-0,187$ & 1 & & \\
\multirow{2}{*}{ BIS-11 } & $-0,234$ & $-0,150$ & 0,310 & 1 & - \\
\hline \multirow{2}{*}{ BHS } & $(0,121)$ & $(0,322)$ & $*(0,03)$ & - & \\
& $0,281(0,061)$ & 0,022 & 0,015 & 0,037 & 1 \\
\hline
\end{tabular}

Tabla 4. Análisis comparativo según sexo.

\begin{tabular}{ccccc} 
& & Hombres $(\mathbf{n}=\mathbf{1 6})$ & Mujeres $(\mathbf{n}=\mathbf{2 9})$ & Valor $\mathbf{p}$ \\
\cline { 2 - 5 } & Edad (años) & $35(26,5-57)$ & $45(32-52)$ & 0,6 \\
\hline Antecedente individual de IS & $68,7 \%(\mathrm{n}=11)$ & $68,9 \%(\mathrm{n}=20)$ & 1 \\
\hline Antecedente familiar de IS & Crónica & $37,5 \%(\mathrm{n}=6)$ & $20,68 \%(\mathrm{n}=6)$ & 0,29 \\
\cline { 2 - 5 } Enfermedad médica & Terminal & $0 \%(\mathrm{n}=0)$ & $3,44 \%(\mathrm{n}=1)$ & 1 \\
\cline { 2 - 5 } & Dolor crónico & $18,75 \%(\mathrm{n}=3)$ & $0 \%(\mathrm{n}=0)$ & $* 0,03$ \\
\cline { 2 - 5 } & Discapacidad & $6,25 \%(\mathrm{n}=1)$ & $6,89 \%(\mathrm{n}=2)$ & 1 \\
\hline & Conflicto familiar & $25 \%(\mathrm{n}=4)$ & $37,93 \%(\mathrm{n}=11)$ & 0,5 \\
\cline { 2 - 5 } Conflicto de pareja & $62,5 \%(\mathrm{n}=10)$ & $58,62 \%(\mathrm{n}=17)$ & 1 \\
\cline { 2 - 5 } Evento estresante & Conflicto laboral & $6,25 \%(\mathrm{n}=1)$ & $3,44 \%(\mathrm{n}=1)$ & 1 \\
\cline { 2 - 5 } & Conflicto económico & $37,5 \%(\mathrm{n}=6)$ & $13,79 \%(\mathrm{n}=4)$ & 0,13 \\
\cline { 2 - 5 } & Duelo & $6,25 \%(\mathrm{n}=1)$ & $17,24 \%(\mathrm{n}=5)$ & 0,39 \\
\cline { 2 - 5 } & Violencia intrafami- & $0 \%(\mathrm{n}=0)$ & $13,79 \%(\mathrm{n}=4)$ & 0,28 \\
\hline Antecedente de trauma psíquico & $25 \%(\mathrm{n}=4)$ & $72,41 \%(\mathrm{n}=21)$ & $* 0,004$ \\
\hline SIS & $17(13,5-22)$ & $12(9-17)$ & 0,08 \\
\hline BLS & $2(0-5,5)$ & $3(2-4)$ & 0,34 \\
\hline BPAQ & $79(70,5-80,5)$ & $76(67-81)$ & 0,34 \\
\hline BIS-11 & $54,5(42,5-72)$ & $60(44-67)$ & 0,75 \\
\hline BHS & $8(5-14)$ & $9(7-15)$ & 0,34 \\
\hline
\end{tabular}

Resultados en medianas (RIQ) o proporciones (número absoluto). Valor p a partir de la prueba exacta de Fisher o la prueba de Wilcoxon-Mann-Whitney. *Diferencias estadísticamente significativas. 
Tabla 5. Análisis comparativo según gravedad.

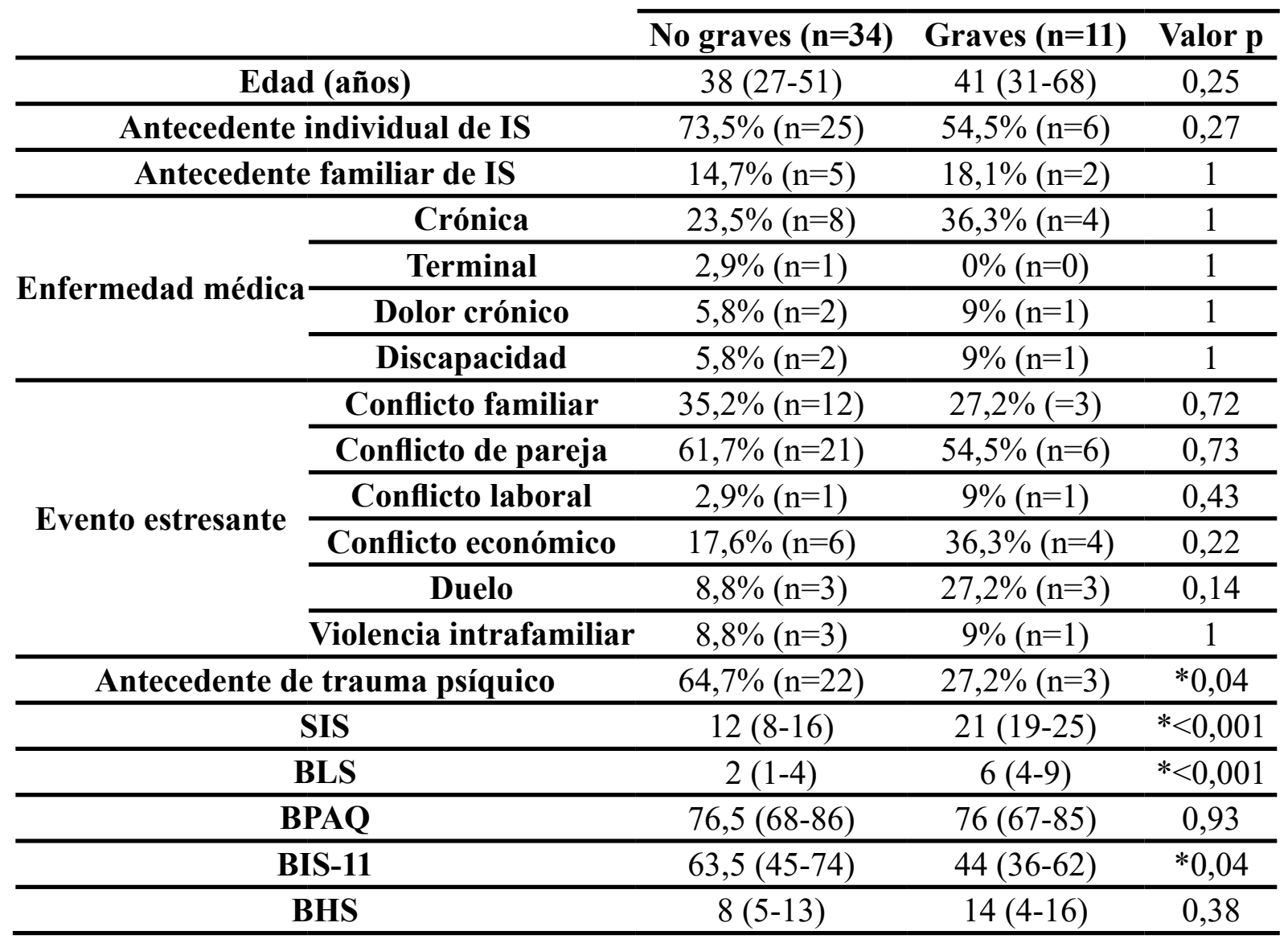

tallará más adelante, los que dificultan la relación armónica con los demás.

Desde el punto de vista socioeconómico, un tercio de la muestra contaba con estudios primarios y $46,6 \%$ con estudios secundarios. La mitad se ubicaba en el segundo quintil de ingresos económicos, es decir, los de menores ingresos. En efecto, solo un tercio tenía un empleo estable. Todo esto redunda en mayores niveles de adversidad-estrés y pocas opciones de vida, lo que aumenta la probabilidad de cometer un IS como forma de resolución del conflicto. En este sentido, un estudio liderado por Näher et $\mathrm{al}^{(35)}$ analizó, según distrito, los datos sociodemográficos de 149.033 suicidios cometidos en Alemania. En cuanto a las tasas de suicidio, encontró un aumento de $1,2 \%$ por cada $1 \%$ de aumento de la cesantía y una disminución de $0,39 \%$ por cada $1 \%$ de incremento en los ingresos económicos, lo que corrobora la relevancia de las condiciones socioeconómicas como determinantes del suicidio.

Todos los participantes de este estudio presentaron al menos un diagnóstico psiquiátrico, siendo el más frecuente el de trastorno de la personalidad, mayormente de estructura limítrofe, en alrededor de $50 \%$, seguido de trastorno por consumo de alcohol $(33,3 \%)$, episodio depresivo mayor $(28,8 \%)$ y trastorno por consumo de otras sustancias $(24,4 \%)$. Hubiese sido plausible hallar una frecuencia mayor de otros diagnósticos que se han asociado clásicamente a la suicidalidad, tales como los trastornos bipolares o la esquizofrenia, pero creemos que esto se vio limitado por el reducido tamaño de la muestra. 
La evaluación de la gravedad del IS puede realizarse en función de los niveles de intencionalidad (SIS) y letalidad médica (BLS). En nuestro estudio se observaron niveles severos de intencionalidad y letalidad en $53,3 \%$ y $44,4 \%$ de la muestra, respectivamente. Esto da cuenta de que el hospital analizado corresponde a un centro de derivación de casos de mayor gravedad de IS. Paralelamente, tanto intencionalidad como letalidad médica se correlacionaron significativamente $(p=0,007)$, lo que reafirma la relevancia de evaluar ambos aspectos durante el manejo de un IS. En este sentido, un reciente estudio prospectivo que incluyó 479 pacientes de una unidad de emergencia psiquiátrica que consultaron por episodios autolesivos, comparó la precisión predictiva de suicidio a un año de la entrevista clínica $y$ de la SIS, sin encontrar diferencias ${ }^{(36)}$. No obstante, este resultado debe evaluarse con cautela, ya que los casos de suicidio incluidos fueron escasos.

No se destacaron altos niveles de impulsividad en esta muestra, ya que alrededor del 80\% mostró niveles nulos o normales. Esto demuestra que, si bien se ha asociado la conducta suicida a la impulsividad, esta dimensión continúa siendo una fuente de discusión ${ }^{(37)}$. En efecto, ciertos autores han descrito una relación paradójica entre impulsividad y letalidad: los IS menos impulsivos han resultado ser más letales ${ }^{(38)}$. Este fenómeno es controvertido, ya que sería igualmente posible afirmar que la letalidad del IS se asocia a menor impulsividad, es decir, a una mayor reflexión en torno al suicidio. Aunque nuestros resultados apoyan esta relación inversa, ya que el coeficiente de correlación entre BIS-11 y BLS fue negativo, la correlación no fue estadísticamente significativa. Sí se halló una correlación positiva y estadísticamente significativa $(p=0,03)$ entre impulsividad y agresividad, lo que es teóricamente esperable. La agresividad mostró una mediana de 76 , sin diferencias significa- tivas según sexo ni gravedad del IS.

Así como la impulsividad, la desesperanza es un concepto dinámico que podría presentarse como estado o como rasgo, siendo este último el que tendría mayor valor predictivo y se asociaría a conductas suicidas $^{(39)}$. Algunas hipótesis han señalado que los constructos desesperanza, pertenencia frustrada (es decir, la percepción de falta de relaciones recíprocas de cuidado y el deseo incumplido de "pertencer") y percepción de carga (es decir, el percibirse como una carga negativa para los demás, lo que mejoraría dejando de existir), interactúan y tributan al deseo suicida, que junto con la capacidad de realizar un IS letal, son los factores claves para el suicidio según ha identificado la teoría interpersonal del suicidio ${ }^{(8)}$. Por lo tanto, sería de gran relevancia explorar las tres dimensiones durante la evaluación de la suicidalidad. Asimismo, la teoría cognitiva del suicidio indica que la desesperanza es la precondición esencial de la ideación suicida en pacientes deprimidos. No obstante, un estudio de cohortes cuestionó este postulado, ya que corroboró que la severidad de los síntomas depresivos predecirían la ideación suicida más precisamente que la desesperanza ${ }^{(9)}$, lo que debe evaluarse en contexto y perspectiva, ya que la desesperanza ha demostrado ser un constructo consistentemente asociado a la suicidalidad. En nuestro estudio, cerca de la mitad de la muestra presentó niveles de desesperanza leve o sin significancia clínica. La desesperanza medida por la BHS correspondería más bien a un fenómeno de estado, en cambio, en la muestra lo más prevalente fue una estructura de personalidad determinada que no reflejaría necesariamente un nivel de desesperanza de forma constante, es decir, como rasgo. En el grupo de casos graves, el diagnóstico más frecuente fue el de episodio depresivo mayor, alcanzando un 54,5\%; asimismo, los niveles de desesperanza en los casos graves fueron superiores, con una mediana de 14 (RIQ 4-16), 
versus una mediana de 8 (RIQ 5-13) en los casos no graves, pero esta diferencia no alcanzó significancia estadística. La mediana de impulsividad fue de 44 (RIQ 36-62), significativamente menor que en los casos no graves, donde se alcanzó una mediana de 63,5 (RIQ 45-74). Esto se condice con el frecuente diagnóstico de depresión unipolar en los casos graves, en donde se confirman niveles altos de desesperanza y bajos de impulsividad, contrario a lo que se observaría en la personalidad límite predominante en el resto de los casos. Conjuntamente, entre los casos más graves se observó que $36,3 \%$ tuvo un deterioro cognitivo leve, lo que se asocia a una menor disponibilidad de recursos cognitivos y afectivos para resolver conflictos psíquicos y vitales. Un hallazgo controvertido fue que la proporción de antecedente de trauma psíquico fue significativamente mayor de entre los casos no graves, toda vez que las investigaciones han documentado el trauma psíquico como un factor de riesgo mayor para $\mathrm{IS}^{(40)}$. Creemos que esto se debió a la baja cantidad de casos graves analizados.

Los resultados presentados deben evaluarse con cautela, pues debido a que el tamaño muestral fue reducido se aplicó es- tadística inferencial no paramétrica, la que solo permite obtener conclusiones con respecto a la muestra, pero no extrapolar resultados a la población de la cual provino; de esta manera, probablemente no fue posible concluir asociaciones teóricamente esperables. Conjuntamente, los diagnósticos dimensionales y categoriales se realizaron mediante pruebas validadas, pero que distan de la profundidad y complejidad diagnóstica que puede lograrse en un contexto clínico y longitudinal.

Se realizó una caracterización exhaustiva y un análisis de variables psicopatológicas y sociodemográficas obtenidas mediante instrumentos de evaluación validados y ampliamente usados en la investigación, destacando diferencias según sexo y gravedad. Esto permitió conocer un perfil biodemográfico y clínico de los pacientes que realizaron un IS en la región, características que han sido poco descritas localmente y que representan a grupos poblacionales en un contexto sociogeográfico particular. Estos hallazgos son relevantes, ya que indican un perfil de pacientes graves que deberían ser pesquisados y manejados en los distintos espacios de atención ambulatoria a nivel intersectorial.

\section{Resumen}

Introducción: El suicidio es una problemática de salud pública global. A nivel regional, el análisis de las dimensiones psicopatológicas y sociodemográficas del intento de suicidio (IS) son escasas. Método: estudio transversal que analizó psicopatológica y sociodemográficamente a adultos hospitalizados por IS en un hospital público de Chile. Los participantes se caracterizaron sociodemográficamente y clínicamente, evaluando síndromes neuropsiquiátricos, trastornos de la personalidad y niveles de desesperanza, impulsividad, intencionalidad, letalidad y agresividad del IS. Se aplicó estadística no paramétrica. Resultados: 45 participantes fueron incluidos, con una mediana de 39 años de edad. La mayoría residió en zonas urbanas, pertenecía a un nivel socioeconómico bajo, eran solteros, presentó algún evento vital estresante y antecedente de IS. El método de IS más frecuente fue la ingesta medicamentosa. Los diagnósticos más frecuentes fueron trastorno por consumo de alcohol y personalidad límite. En la mitad de los casos la desesperanza fue leve o inexistente. 
La letalidad se correlacionó positiva y significativamente con la intencionalidad suicida, así como la impulsividad con la agresividad. El antecedente familiar de IS y el trauma psíquico fueron significativamente mayores en mujeres y el dolor crónico en hombres. Los casos graves mostraron altos niveles de desesperanza, intencionalidad y letalidad, mientras que la impulsividad fue significativamente menor que en los casos no graves. En este grupo, el diagnóstico más frecuente fue episodio depresivo. Conclusiones: La muestra analizada mostró características psicopatológicas y sociodemográficas distintivas. La letalidad y la intencionalidad del IS deben ser sistemáticamente evaluadas. Los casos graves de IS mostraron características psicopatológicas diferenciales.

Palabras clave: suicidio, psicopatología, adulto, hospitalización

\section{Referencias Bibliográficas}

1.- Bachmann S. Epidemiology of suicide and the psychiatric perspective. Int J Env Res Public Heal. 2018;15(7):E1425.

2.- World Health Organization. Preventing suicide: A global imperative. Geneva; 2014.

3.- $\quad$ Servicio de Salud Viña del Mar - Quillota. Base de egresos hospitalarios 2014. Viña del Mar; 2014.

4.- Servicio de Salud Viña del Mar - Quillota. Registro de los Servicios de Urgencia Hospitalarios 2015. Viña del Mar; 2015.

5.- Turecki G, Brent DA. Suicide and suicidal behaviour. Lancet. 2016;387(10024):1227-39.

6.- Nock MK, Borges G, Bromet EJ, Cha CB, Kessler RC, Lee S. Suicide and suicidal behavior. Epidemiol Rev. 2008;30(1):133-54.

7.- Knorr AC, Ammerman BA, Hileman B, Kraus C, Nemoianu A, Strony R. 50 influential predictors of suicide attempt among suicidal ideators following an emergency department visit. Ann Emerg Med. 2018;72(4):S23.
8.- Tucker RP, Hagan CR, Hill RM, Slish ML, Bagge CL, Joiner TE, et al. Empirical extension of the interpersonal theory of suicide: Investigating the role of interpersonal hopelessness. Psychiatry Res. 2018;259:427-32.

9.- Baryshnikov I, Rosenström T, Jylhä P, Vuorilehto M, Holma M, Holma I, et al. Role of hopelessness in suicidal ideation among patients with depressive disorders. J Clin Psychiatry. 2020;81(2).

10.- Rath D, de Beurs D, Hallensleben N, Spangenberg L, Glaesmer H, Forkmann T. Modelling suicide ideation from beep to beep: Application of network analysis to ecological momentary assessment data. Internet Interv. 2019;18:100292.

11.- Cole AB, Littlefield AK, Gauthier JM, Bagge CL. Impulsivity facets and perceived likelihood of future suicide attempt among patients who recently attempted suicide. J Affect Disord. 2019;257:195-9.

12.- Miller IW, Camargo CA, Arias SA, Sullivan AF, Allen MH, Goldstein AB, et al. Suicide prevention in an emergency department population: The 
ED-SAFE study. JAMA Psychiatry. 2017;74(6):563-70.

13.- Beautrais AL. Further suicidal behavior among medically serious suicide attempters. Suicide Life Threat Behav. 2004;34(1):1-11.

14.- Bowers A, Meyer C, Hillier S, Blubaugh M, Roepke B, Farabough M, et al. Suicide risk assessment in the emergency department: Are there any tools in the pipeline? Am J Emerg Med. 2018;36(4):630-6.

15.- Silverman MM, Berman AL, Sanddal ND, O'Carroll PW, Joiner TE. Rebuilding the tower of Babel: A revised nomenclature for the study of suicide and suicidal behaviors. Part 2: Suicide-related ideations, communications, and behaviors. Suicide Life Threat Behav. 2007;37(3):264-77.

16.- Beck A, Schuyler D, Herman I. Development of suicidal intent scales. In: Beck A, Resnik H, Lettieri D, editors. The Prediction of Suicide. First Edit. Philadelphia: Charles Press; 1974. p. 45-6.

17.- Beck AT, Beck R, Kovacs M. Classification of suicidal behaviors: I. Quantifying intent and medical lethality. Am J Psychiatry. 1975 Mar;132(3):285-7.

18.- Buss AH, Perry M. The Aggression Questionnaire. J Pers Soc Psychol. 1992;63(3):452-9.

19.- Rodríguez J, Peña E, Graña J. Adaptación psicométrica de la versión española del Cuestionario de Agresión. Psicothema. 2002;14(2):476-82.

20.- Morales-Vives F, Codorniu-Raga MJ, Vigil-Colet A. Características psicométricas de las versiones reducidas del cuestionario de agresividad de Buss y Perry. Psicothema. 2005;17(1):96100.

21.- Barratt E. Impulsiveness and Aggression. In: Monahan J, Steadman H, editors. Violence and Mental Disor- der Development in risk assessment. Chicago: The University of Chicago Press; 1995. p. 61-79.

22.- Oquendo M, Baca-García E, Graver R, Morales M, Montalbán V, Mann J. Spanish adaptation of the Barratt Impulsiveness Scale (BIS-11). Eur J Psychiatry. 2001;15(3):147-55.

23.- Beck AT, Weissman A, Lester D, Trexler L. The measurement of pessimism: The Hopelessness Scale. J Consult Clin Psychol. 1974;42(6):861-5.

24.- Rueda-Jaimes GE, Castro-Rueda VA, Rangel-Martínez-Villalba AM, Moreno-Quijano C, Martinez-Salazar GA, Camacho PA. Validación de la Escala de Desesperanza de Beck en pacientes con riesgo suicida. Rev Psiquiatr Salud Ment. 2018;11(2):86-93.

25.- Nasreddine Z, Phillips N, Bédirian V, Charbonneau S, Whitehead V, Collin I, et al. The Montreal Cognitive Assessment, MoCA: a brief screening tool for mild cognitive impairment. J Am Geriatr Soc. 2005;53(4):695-9.

26.- Delgado C, Araneda A, Behrens M. Validation of the Spanish-language version of the Montreal Cognitive Assessment test in adults older than 60 years. Neurologia. 2019;34(6):37685.

27.- Ferrando L, Bobes J, Gibert J, Franco-Alonzo L, Soto O, Franco L. MINI International Neuropsychiatric Interview. Versión en español 5.0.0. DSMIV. Madrid; 1998.

28.- AW L, Sartorious N, Andreoli A, Berger P, Buchheim P, Channabasavanna $\mathrm{S}$, et al. The International Personality Disorder Examination. Arch Gen Psychiatry. 1994;51(3):215-24.

29.- López-Ibor J, Pérez A, Rubio V. Examen Internacional de los Trastonos de la Personalidad (IPDE): Módulo DSM-IV y CIE-10. Madrid: Meditor; 1996. 
30.- von Elm E, Altman DG, Egger M, Pocock SJ, Gøtzsche PC, Vandenbroucke JP, et al. The Strengthening the Reporting of Observational Studies in Epidemiology (STROBE) statement: Guidelines for reporting observational studies. PLoS Med. 2007;4(10):e296.

31.- Borges G, Nock MK, Abad JMH, Hwang I, Sampson NA, Alonso J, et al. Twelve-month prevalence of and risk factors for suicide attempts in the world health organization world mental health surveys. J Clin Psychiatry. 2010;71(12):1617-28.

32.- Tsirigotis K, Gruszczynski W, Tsirigotis M. Gender differentiation in methods of suicide attempts. Med Sci Monit. 2011;17(8):PH65.

33.- Akbarizadeh F, Hajivandi A, Hajivandi M, Zeidabadinejad MS. Marriage related suicide fatality rates. Iran J Psychiatry. 2019;14(1):54-9.

34.- Fukuchi N, Kakizaki M, Sugawara Y, Tanji F, Watanabe I, Fukao A, et al. Association of marital status with the incidence of suicide: A population-based cohort study in Japan (Miyagi cohort study). J Affect Disord. 2013;150(3):879-85.

35.- Näher A-F, Rummel-Kluge C, Hegerl $\mathrm{U}$. Associations of suicide rates with socioeconomic status and social isolation: Findings from longitudinal register and census data. Front Psychiatry. 2019; 10:898.

36.- Lindh ÅU, Beckman K, Carlborg A, Waern M, Salander Renberg E, Dahlin $\mathrm{M}$, et al. Predicting suicide: A comparison between clinical suicide risk assessment and the Suicide Intent Scale. J Affect Disord. 2020;263:445-9.

37.- Baca-García E, Diaz-Sastre C, García Resa E, Blasco H, Braquehais Conesa D, Oquendo MA, et al. Suicide attempts and impulsivity. Eur Arch Psychiatry Clin Neurosci. 2005;255(2):152-6.
38.- Baca-García E, Diaz-Sastre C, Basurte E, Prieto R, Ceverino A, Saiz-Ruiz J, et al. A prospective study of the paradoxical relationship between impulsivity and lethality of suicide attempts. J Clin Psychiatry. 2001 Jul;62(7):560-4.

39.- Young M, Fogg L, Scheftner W, Fawcett J, Akiskal H, Maser J. Stable trait components of hopelessness: Baseline and sensitivity to depression. J Abnorm Psychol. 1996;105(2):155-65.

40.- Chang EC, Lucas AG, Chang OD, Duan T, Zhou Z, Yang JZ, et al. Presence of trauma and suicide risk: Personal control as a moderator. Death Stud. 2018;42(8):529-33.

Correspondencia:

Reginald Rees

Departamento de Psiquiatría

Universidad de Valparaíso,

Subida Carvallo 200, Casilla 92-V,

Valparaíso, Chile

reginald.rees@uv.cl 